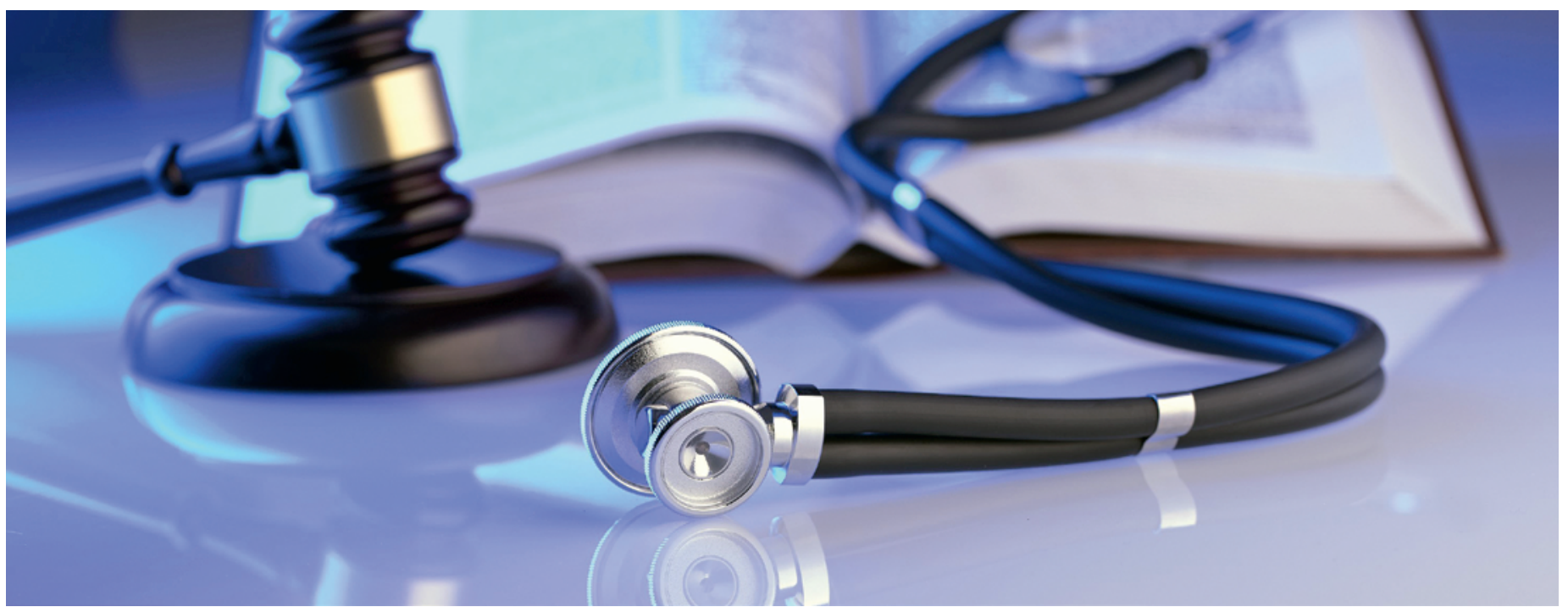

\title{
Neues Arzthaftungsmodul der FMH und SIM für medizinische Gutachter
}

\section{Iris Herzog-Zwitter}

Dr. iur., Juristin FMH-Rechtsdienst, SIM Bildungsbeauftragte

\section{Arzthaftungsmodul FMH / SIM für Gutachter}

Die Entwicklungen im Arzthaftungsrecht bringen eine gesteigerte Nachfrage an Gutachten mit sich. Die FMH und die Swiss Insurance Medicine (SIM) haben gemeinsam neu ein Arzthaftungsmodul für Mediziner erarbeitet. Ziel des Moduls ist es, dieser Nachfrage gerecht $\mathrm{zu}$ werden und die Gutachterqualität zu steigern, indem die Gutachter spezifisch für ihre Gutachtertätigkeit und für die fachlichen Brennpunkte des Arzthaftungsrechts ausgebildet werden. Die juristische Terminologie des Arzthaftungsrechts ist im konkreten Fall untrennbar mit der gutachterlichen Tätigkeit verbunden.

\section{In einem Haftpflichtprozess ist immer die Qualität des Gutachtens massgebend.}

Die aktuelle Rechtsentwicklung und Gesetzgebungsverfahren im Medizinrecht, welche Einfluss auf die Entwicklung im Arzthaftungsrecht haben, finden in das Bildungskonzept dieses Arzthaftungsmoduls Eingang. Zudem ist es ein Faktum, dass das Recht der Medizin und der Technik hinterherhinkt. Diesem Umstand wurde auch bei der Entwicklung dieses Moduls Rechnung getragen.
Das vorliegende Ausbildungsmodul bietet Medizinern die Möglichkeit, sich in der Rechtsmaterie des Arzthaftungsrechts weiterzubilden. Zielgruppe sind Mediziner, die in streitigen Arzthaftungsfällen zur Frage der ärztlichen Sorgfaltspflicht Gutachten erstellen. Insbesondere die Beurteilung des Behandlungsfehlers im versicherungsmedizinischen Kontext ist einer der Brennpunkte der Gutachtertätigkeit. Eine grosse fachliche Herausforderung in jedem Gutachten ist zum Beispiel die Beurteilung, ob ein Behandlungsfehler zum Zeitpunkt der Behandlung vorliegt und wie die systematische Einordnung des Behandlungsfehlers zu erfolgen hat.

In einem Haftpflichtprozess ist immer die Qualität des Gutachtens massgebend bzw. ob ein Gutachten in einem Haftpflichtprozess als verwertbares Gutachten bzw. Beweismittel im versicherungsmedizinischen Kontext erachtet wird. Die Qualität der Gutachten ist im engen Kontext mit der Rechtssicherheit und Patientensicherheit zu sehen. Die bundesgerichtliche Rechtsprechung definiert, was von einem beauftragten Gutachter erwartet werden darf: Der Gutachter hat den Auftrag aufgrund seiner berufsspezifischen Sachkunde mit der gebotenen vertragsmässig geschuldeten Sorgfalt auszuführen. Die Ausführungen eines Sachverständigen sind gemäss Rechtsprechung bei Arzthaftungsprozessen auf Vollständigkeit und Wider- 
sprüchlichkeit zu prüfen. Gemäss bundesgerichtlicher Rechtsprechung wird Privatexpertisen unter bestimmten Voraussetzungen wie einem FMH-Gutachten «durchaus volle Beweiskraft zuerkannt» [1].

\section{Pilotprojekt}

Am 22./23. Januar 2020 wurde an zwei Abenden das Pilotprojekt im Universitätsspital Basel durchgeführt. Mediziner und Juristen referierten zur Arzthaftung und Gutachtertätigkeit. Die Pilotdurchführung fand in deutscher Sprache in Seminargrösse statt.

Nebst den Parameterthemen der Arzthaftung «die ärztliche Sorgfaltspflicht, die ärztliche Aufklärungspflicht und die ärztliche Dokumentationspflicht» wurde über den Begriff der «Kausalität» und «beweisrechtliche Fragen» doziert. Diese Fragen beschäftigen nicht nur den Gutachter bei der Erstellung eines Gutachtens, obwohl es sich um eine juristische Frage handelt, sondern jeden Arzt bei der Behandlung von Patienten. Letztendlich ist die natürliche Kausalität mit dem Beweisgrad der überwiegenden Wahrscheinlichkeit das entscheidende Kriterium, um eine Haftung zu bejahen. Das Bundesgericht hat mittels Rechtsprechung präzisiert, wann der Beweisgrad der überwiegenden Wahrscheinlichkeit gegeben ist. Weitere zentrale Punkte waren das FMH-Gutachten und das revidierte FMH-Ablaufschema sowie das Gemeinschaftliche Gutachterkonsilium (GGK) [2]. Mit dem neuen Reglement der FMH-Gutachterstelle wird zusätzlich zum schriftlichen Begutachtungsverfahren optional das FMH-GKK als mündliche Besprechung am runden Tisch eingeführt. Das GGK wurde an den Basler Arzthaftpflichttagen 2012 entwickelt und wird von der asim (Academy of Swiss Insurance Medicine/Universitätsspital Basel) umgesetzt. Es stellt ein flexibles Modell für eine aussergerichtliche, mündliche Abarbeitung der medizinischen Fragestellungen in Arzthaftpflichtfällen dar unter direktem Einbezug aller Beteiligten und Betroffenen.

\section{Das Pilotprojekt wurde im Januar im Uni- versitätsspital Basel durchgeführt.}

Die von den teilnehmenden Medizinern eingegebenen Fragen zur "Arzthaftung und Gutachtertätigkeit» wurden mit Bezug zur bundesgerichtlichen Rechtsprechung diskutiert. Ebenso fand das sensible Thema «Kommunikation bei einem vermuteten Behandlungsfehler» Eingang in die gemeinsame Diskussion.

\section{Bildnachweis}

(c) Franky44 | Dreamstime.com

\section{Literatur}

1 Landolt/Herzog-Zwitter, Arzthaftungsrecht, S. 277.

2 https://www.unispital-basel.ch/ueber-uns/bereiche/medizin/ kliniken-institute-abteilungen/asim-versicherungsmedizin/asim begutachtung/erfassungstool-ggk/ (letzter Zugriff am 14.4.2020)

\section{Vom Pilotprojekt zum festen Arzthaftungsmodul}

Das nächste Arzthaftungsmodul wird am 19. August 2020 in Zürich in der Klinik Hirslanden durchgeführt. Aufgrund der Auswertung der Evaluationsergebnisse des Pilotprojektes ist die Durchführung neu an einem Tag von 15.00 bis 21.30 Uhr inklusive Verpflegungspausen vorgesehen. Die Durchführung findet in deutscher Sprache statt, der französische Lehrgang des Arzthaftungsmoduls für das Jahr 2020 ist derzeit in Planung.

Der inhaltliche Aufbau basiert auf den positiven Evaluationsergebnissen des Pilotprojektes. Zusätzlich werden Themen wie Fehler und "No-Gos» in den Formulierungen, Brennpunkte der medizinischen Notfallversorgung und rechtliche Probleme im Zusam- menhang mit der Vertretermedizin in das Programm integriert. Im Rahmen dieses Arzthaftungsmoduls wird dem Austausch und der gegenseitigen Einschätzung der Mediziner zu den Stolpersteinen der Arzthaftung zusätzliche Priorität eingeräumt.

In der Kursgebühr von CHF 500 ist die Teilnahmebestätigung und Verpflegung inbegriffen. Das SIWF und die SIM vergeben je 6 Credits für die Teilnahme am Arzthaftungsmodul.

Weitere Informationen zur Durchführung und Anmeldung für das nächste Arzthaftungsmodul finden Sie unter: www.fmh.ch/gutachterausbildung-arzthaftung 